\title{
Effects of Wind Turbine Generators on Inter-Area Oscillations and Damping Control Design
}

\author{
Felipe Wilches-Bernal \\ Sandia National \\ Laboratories \\ fwilche@sandia.gov
}

\author{
Christoph Lackner \\ Rensselaer Polytechnic \\ Institute \\ lacknc@rpi.edu
}

\author{
Joe H. Chow \\ Rensselaer Polytechnic \\ Institute \\ chowj@rpi.edu
}

\author{
Juan J. Sanchez-Gasca \\ GE \\ Energy \\ juan1.sanchez@ge.com
}

\begin{abstract}
This paper analyzes the effect of wind turbine integration (WT) on the inter-area oscillation mode of a test two-area power system. The paper uses a root-locus based design method to propose a pair of controllers to provide damping to the inter-area mode of the system. The controllers are selected from the best combination of feedback signal and WT control action. One of the controllers uses the active power control part of the WT while the other uses the reactive power part. The paper analyzes the impact that increases on the transmission line connecting the WT to the system have on the controllers' performance. Time domain simulations are provided to evaluate the effectiveness of the controllers under different conditions.
\end{abstract}

\section{Introduction}

The future grid is expected to accommodate much higher levels of penetration of wind and solar generation if goals such as the 50\% mandate of electricity coming from renewables by 2030 in states like California and New York are to be met $[1,2]$. Because of this continuous introduction of renewable energy the traditional operation of power systems is expected to change. It is important to assess how the inclusion of converter-based interfaced clean energy sources, such as wind and solar, affect the dynamics of power systems traditionally set by the swing equation governing conventional generators. In particular, studying the impact that power electronics interfaced generation has on the small signal stability of power system is critical to

Sandia National Laboratories is a multimission laboratory managed and operated by National Technology and Engineering Solutions of Sandia, LLC., a wholly owned subsidiary of Honeywell International, Inc., for the U.S. Department of Energy's National Nuclear Security Administration under contract DE-NA0003525.

This research was supported in part by the U.S. Department of Energy Transmission Reliability program.

This work was supported in part by the Engineering Research Center Program of the National Science Foundation and the Department of Energy under NSF Award Number EEC-1041877 and the CURENT Industry Partnership Program. enable its inclusion in larger quantities. Because power electronics have much faster responses than conventional generation and can independently control active and reactive power it is of special interest to investigate how they can help with the small signal stability of the system.

The effect that integrating Wind Turbine Generators (WTGs) has on inter-area oscillations have been investigated in [3-8]. Most of these studies show that wind integration increases the damping of these oscillations. However, some works show that under certain operating conditions the installation of wind generation can destabilize these modes of oscillation [5, 9]. Previous research has shown power electronics interfaced generation to be effective in damping power system oscillations [6,10-14].

This paper is composed of two main parts. The first studies the effect of integrating WTG in a two-area system representative of a transfer path continuing the work presented in $[15,16]$. The second part proposes root-locus control strategy for WTGs to damp inter-area oscillations. The linear analysis and nonlinear simulation of wind integration presented in this paper are facilitated by the MATLAB-based Power System Toolbox (PST) software [17] using the implemented Type-3 WTG model $[7,18]$. The main contributions of this work can be stated as follows:

(a) It studies the effects on the inter-area mode of the system of increasing wind penetration for different power transfer conditions. It shows that integrating WTGs in the area that is importing power may pose small signal stability problems.

(b) It presents an investigation on how the transmission line used to perform the installation of wind in the system affect its inter-area mode.

(c) It proposes two different control mechanisms for WTG to damp inter-area oscillations. One controller actuates in the active power control of the WTG while the other is included in the reactive power control.

(d) It studies the proper combination of measured signal 
and point of connection of the controller within the WTG to better influence the inter-area mode of the system.

(e) It analyzes the effect of transmission line increases on the proposed controllers. It shows that even though increases in the transmission line only affect slightly the inter-area mode of the system, the zeros of the system are heavily affected. The movement of the zeros is shown to decrease noticeably the performance of both proposed controllers.

(f) Time domain simulation is performed to show that WTG can be effectively used as a tool to damp inter-area oscillations and different input/output signal combinations can be used to achieve that goal.

\section{Test System and WTG Model}

\subsection{WTG Model}

The Type-3 WTG model presented in $[19,20]$ and implemented in PST [18] is used in this work. The model can represent up to an entire wind power plant and has been previously validated [21-25]. The converter-controlled grid interface of this type WTG allows for independent active and reactive power control. The model interacts with the grid as a controlled current injection. The injected current is determined by the outputs of the active and reactive power controllers, $I_{p \mathrm{cmd}}$ and $E_{f d \mathrm{cmd}}$ respectively as well as the voltage at the point of interconnection. The current injected by the Type-3 WTG model is

$$
I_{t}=\left(I_{p}-j \frac{E_{f d}}{L_{p p}}\right)(\cos (\gamma)+j \sin (\gamma))+j \frac{\tilde{V}_{t}}{L_{p p}}
$$

where $I_{p}$ and $E_{f d}$ correspond to the outputs of the active and reactive power controllers after they pass through an independent delaying stage each. $\tilde{V}_{t}$ is the voltage at the point where the WTG is connected and $\gamma$ is a state representing the PLL action of the converter [20] that is described by

$$
\dot{\gamma}=k_{p l l p}\left[V_{t \mathrm{im}} \cos (\gamma)-V_{t \mathrm{re}} \sin (\gamma)\right]
$$

\subsection{Test System}

The test power system used in this paper is presented in Fig. 1. This system corresponds to a two-area, two-machine test system that contains a dominant inter-area mode and can be interpreted as an example of a transfer path between sparse areas that oscillate against each another [26]. To study the effect that positioning of wind generation has on the inter-area power swing of this system, WTGs are to be located independently in each area and at the midpoint of the transfer path. As such three cases are considered:
- Case A - Wind integration is performed in Area 1 at Bus 6. The power output of the conventional generator at Bus 2 is reduced according to the desired wind penetration.

- Case B - Wind integration is performed in Area 2 at Bus 10. The power output of the conventional generator at Bus 4 is reduced according to the desired wind penetration.

- Case C - Wind integration is performed at the transfer path's midpoint at Bus 8. Both conventional machines, generators at Buses 2 and 4 are each reduced by half of the power produced by the WTG.

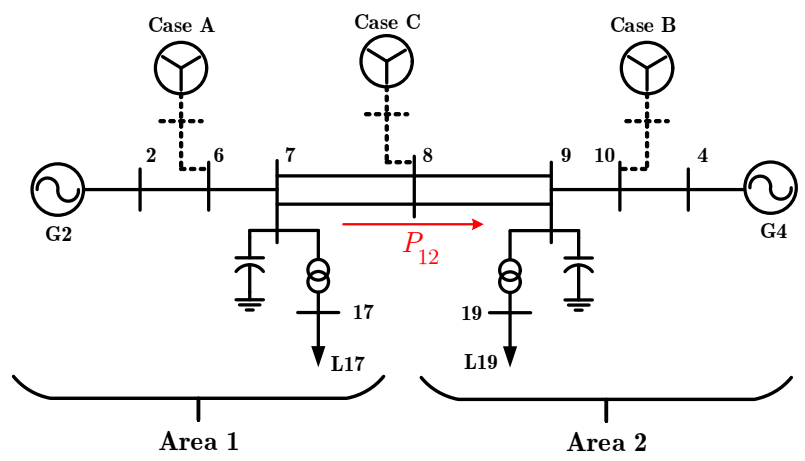

Figure 1: Two-area, two-machine test system used in this paper that has a strong inter-area oscillation mode.

Note that in each case only one WTG is considered as the idea is to see how individual WTG controls interact with the modes of the system. In addition to the cases of WTG positioning, two scenarios regarding how the displacement of conventional generation is achieved are investigated:

- Scenario 1 - Implies a reduction on the MVA of the conventional machine displaced by wind integration according to the penetration level.

- Scenario 2 -The MVA of the conventional machine displaced by wind integration remains constant and only its power level is adjusted according to wind penetration.

Scenario 1 is useful to study long-term impact of wind integration when installation of new wind power plants is coupled with the decommissioning of conventional machines. Scenario 2 is meant to represent situations when wind power plants are producing high amounts of power ${ }^{1}$ and conventional generation is kept as reserve.

\footnotetext{
${ }^{1}$ Higher than the average possibly closer to their nominal rating because of strong wind.
} 


\section{Impact of WTG on Inter-Area Oscillations}

The impact that Type-3 WTG installation has on the test system of Fig. 1 is presented in this section.

\subsection{Inter-area Mode Variations with Increased Wind Penetration}
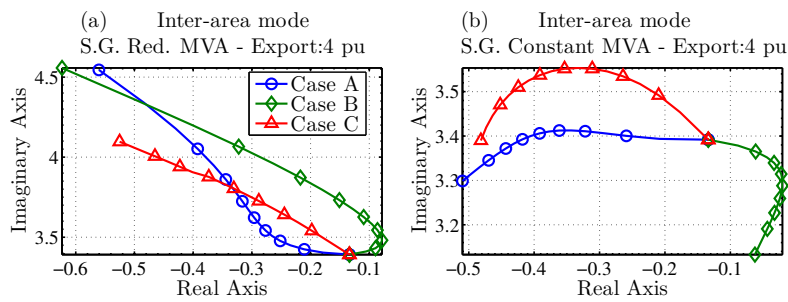

(c) Inter-area mode

S.G. Red. MVA - Export:0 pu
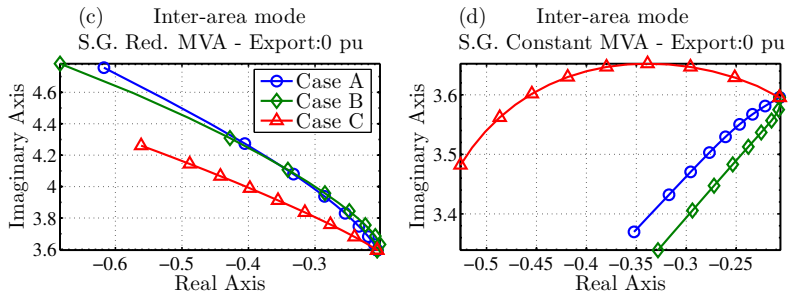

(e) Inter-area mode
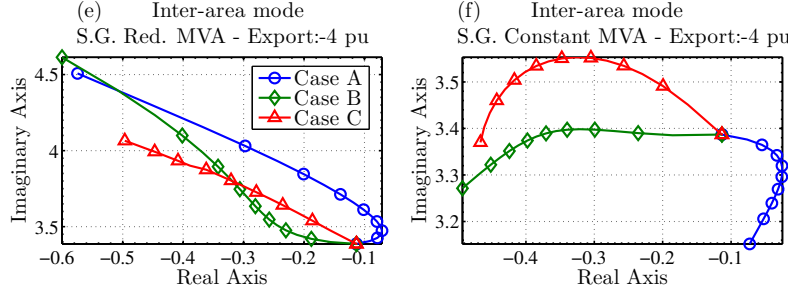

Figure 2: Variations in the inter-area mode of the test system with increments in wind penetration.

A study on the effect that increments of wind penetration has on the inter-area oscillation of the test system is presented in this section. The study is carried out with variations of wind penetration from $0 \%$ to $43 \%$ of the total power production. The three different cases of positioning of wind integration along with the 2 scenarios of displacement of conventional generation outlined in Section 2.2 are considered. In addition, the analysis is performed at three different power transfer conditions: $400 \mathrm{MW}$ (4 pu) export, no power exchange, and $400 \mathrm{MW}$ (4 pu) import.

Fig. 2 shows the variations in the inter-area mode of the system for the 18 different configurations described above. The results in Figs. 2 (a),(c),(e) show the movement of the inter-area oscillation for Scenario 1 where the MVA of the displaced conventional machine is rescaled. The results in Figs. 2 (b),(d),(f) correspond to Scenario 2 of synchronous machine displacement. The three rows on Fig. 2 correspond to the result for the three different power transfer conditions. The results of increasing wind penetration presented in Fig. 2 show
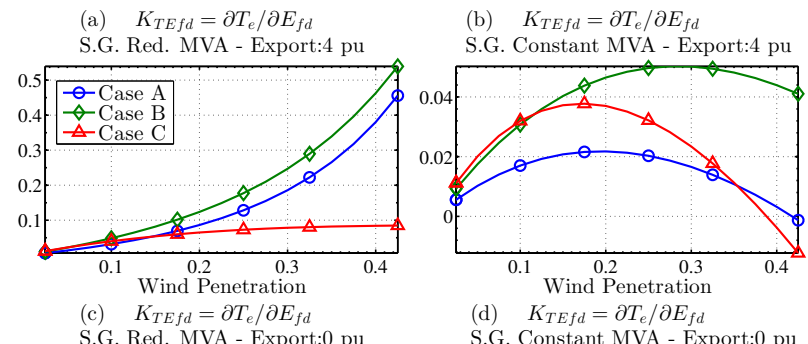

S.G. Red. MVA - Export:0 pu

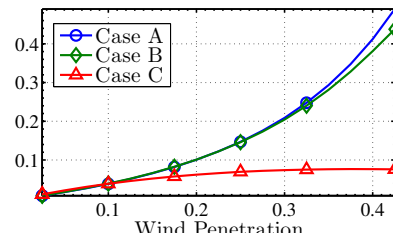

d) $K_{T E f d}=\partial T_{e} / \partial E_{f d}$

S.G. Constant MVA - Export:0 pu

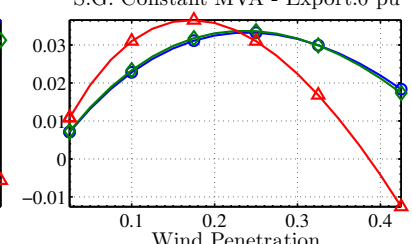

(e) $K_{T E f d}=\partial T_{e} / \partial E_{f d}$

S.G. Red. MVA - Export:-4 pu

(f) $\quad K_{T E f d}=\partial T_{e} / \partial E_{f d}$

(f) $\quad K_{T E f d}=\partial T_{e} / \partial E_{f d}$
S.G. Constant MVA - Export:-4 pu
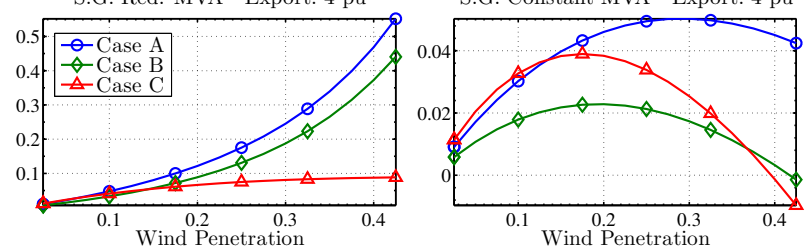

Figure 3: Electrical modal torque of the system as affected by the reactive power current command of the WTG.

that:

- For the cases when installing WTGs causes a reduction in the MVA rating of the displaced conventional machine (Figs. 2(a),(c),(e)), an increase in wind penetration leads to an increase in both, synchronizing and damping torques.

- For cases A and B of wind integration positioning with small penetration (green line in Figs. 2(a) and blue line in Figs. 2(c)) it can be noted that the damping control is initially reduced before it is increased. That is for smaller wind penetration conditions the loading condition of the system affects the impact of wind integration on the damping torque.

- The conditions presented in Figs. 2(b), (f), (cases of Scenario 1 for constant MVA rating in the displaced conventional generator) show that installing WTGs in the area that is importing power reduces the value of the damping and synchronizing torques of the inter-area mode of the system.

Fig. 3 shows the sensitivity $K_{T E f d}$ as a function of increments in wind penetration. This sensitivity is found from the linearized model using $K_{T E f d}=$ $\partial T_{e} / \partial E_{\mathrm{fdcmd}}$ and a more detailed derivation can be found in [15]. The cases considered in this analysis 
correspond to the same ones considered for the study in Fig. 2. These results indicate that higher values of $K_{T E f d}$ have a destabilizing effect on the damping torque of the mode. They are also aligned with results in previous work [4,5]. As discussed in [15] the difference between the two conventional machine displacement scenarios can be attributed to differences in the inertia present on the system.

\subsection{Inter-area Mode Variations with WTG Connecting Transmission Line}

Hitherto, the installation of WTG in the system is made through a substation transformer and a transmission line (TL) that connects it to Bus 6,10 or 8 for Cases A, B or C respectively. In this section the effects of increasing the reactance of the TL that connects the WTG to the system are assessed. The reactance was varied from 0 to $0.11 \mathrm{pu}$ which is equivalent to a transmission line length from 0 to 110 $\mathrm{km}$. The loading condition for the analysis was an import of $400 \mathrm{MW}$ in Area 1 from Area 2. Fig. 4 shows the movement of the inter-area oscillation of the system for all the cases and scenarios of Section 2.2. These results can be described as follows:

- For all the cases of wind positioning when the MVA rating of the displaced machine is reduced (Scenario 1) there is a decrease of the synchronizing torque as the WTG reactance is increased.

- The damping torque increases for Case A which is the area that is importing power. For cases B and $\mathrm{C}$ there is a minor decrease in the damping torque.

- For Scenario 2 when the MVA rating of the displaced synchronous generator remains constant there is a reduced effect on how the mode is affected for Cases A and B with respect to those cases in Scenario 1. However the trends are the same, a reduction on the synchronizing torque for both cases and an increase in the damping torque for Case B and reduction of it for case A. For case C the effect in Scenario 2 is similar to Scenario 1, which represents a considerable reduction in both damping and synchronizing torques.

\section{WTG Damping Control Design}

This section presents a systematic approach to design an inter-area oscillation damping controller for WTGs. The presented approach analyzes a set of signals to determine the most suitable one to use as a feedback input for the controller. The considered signals are as follows:
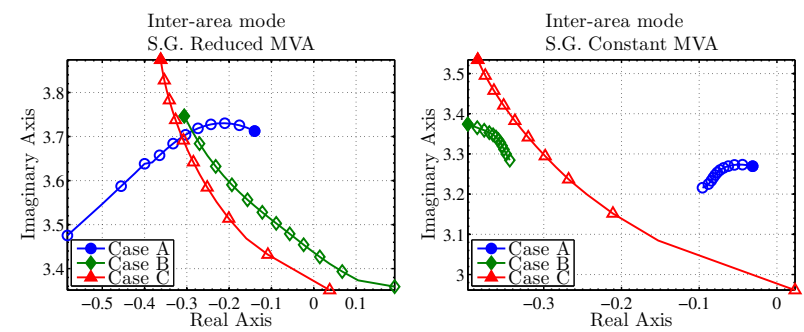

Figure 4: Inter-area mode variations with increases in the reactance that connects the WTG to the system.

- Frequency at the point of interconnection of the WTG.

- Voltage magnitude at the point of interconnection of the WTG.

- Magnitude of the current flowing from Area 1 to Area 2, measured on one of the lines connecting Buses 7 and 8.

- Active power flow from Area 1 to Area 2, measured on one of the lines connecting Buses 7 and 8 .

In combination to the feedback signal selection, this work evaluates the proper place to integrate the controller within the WTG to enhance its ability to influence the power oscillation. Three different WTG points of action considered are:

- Adding a signal $P_{\text {ordsig }}$ to $P_{\text {ord }}$ right before the output of the active power control of the WTG as seen in Fig. 5.

- Adding a signal $P_{\text {setsig }}$ at the active power setpoint control of the WTG as observed in Fig. 5. It should be noted that this action implies the WTG active power output is curtailed or the WTG is spilling wind and has the ability to increase it's active power output.

- Including a signal $Q_{\mathrm{Qsig}}$ inside the WTG reactive power control as shown in Fig. 5.

The power system loading condition selected to perform the controller design is when $4 \mathrm{pu}$ of real power are being imported by Area 1 and the WTG is installed in Area 1 (case A). The WTG displacing half of the conventional generation in the area but the MVA rating of the substituted machine is retained. This case was chosen because this is one where wind integration creates a destabilizing effect for the power oscillation of the system. 


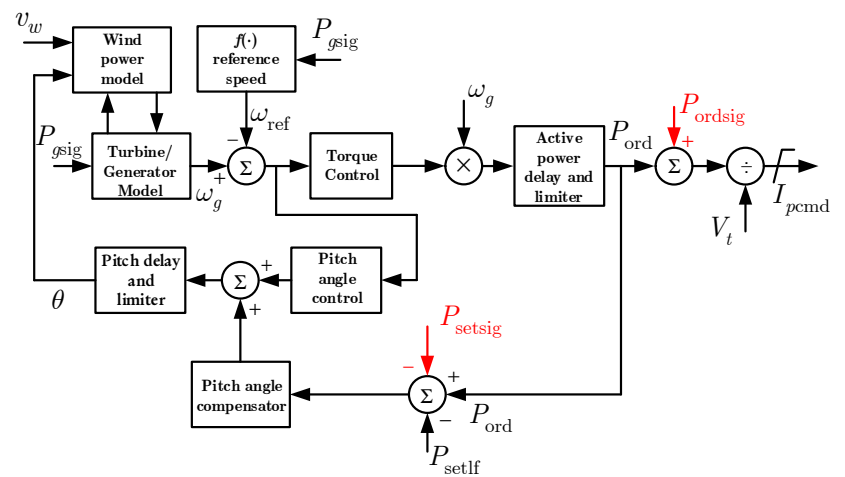

Figure 5: Schematic diagram of the active power control of the WTG model.

\subsection{General Concepts}

The control approach taken in this paper requires a linearized representation of the power system in the following state-space form

$$
\dot{x}=A x+B_{\mathrm{zz}} u, \quad y=C_{\mathrm{xx}} x
$$

obtained at an equilibrium point which corresponds to the power system loadflow.

Since different measured signals and points of action of the controller are being considered different input and ouput matrices are calculated to that end. In particular, three different input matrices $B_{\mathrm{QQ}}, B_{\text {Pset }}$, $B_{\text {Pord }}$ are computed to consider the three different points of connection of the controller mentioned earlier. Similarly, four output matrices, $C_{\mathrm{f}}, C_{\mathrm{V}}, C_{\mathrm{Il}}, C_{\mathrm{P}}$ are obtained to account for the different feedback signal selection. As such any combination of one input matrix $B_{\mathrm{zz}}$ with any output matrix $C_{\mathrm{xx}}$ yields a different system $H_{\mathrm{xx}, \mathrm{zz}}(s)$ to analyze. Note that any particular system is described by 47 states out of which 17 correspond to the WTG.

Based on the close relationship between root-locus analysis and phase compensator design presented in [2730] for PSS tuning, this paper will use root-locus diagrams for the controller design. In the proposed approach, the angle of departure of the inter-area oscillation is determined. Because the idea is to create an increase in damping torque, the proposed controller would require the inter-area mode to move towards the LHP as seen in Fig. 6.

The controller structure utilized in this work is depicted in Fig. 7 and is similar to the structure of PSSs included in conventional generation. Because the controller needs to be inactive for steady state it has a washout filter (derivative action). In addition, it contains two lead-lag compensator stages to move the departure of the mode in the direction of increased damping torque.

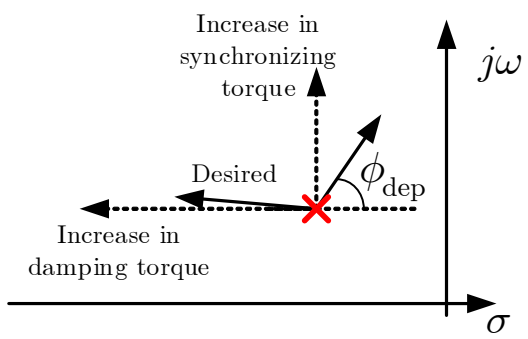

Figure 6: Controller design objective: increase the damping torque of the Inter-Area Mode.

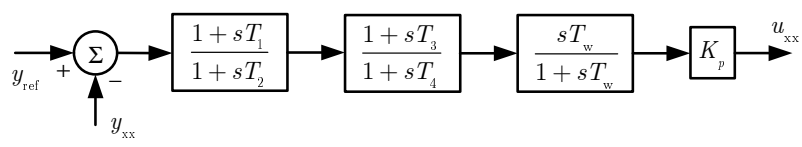

Figure 7: Proposed controller structure. A two-stage lead lag compensator with a washout filter.

\subsection{WTG Active and Reactive Power Controls}

Fig. 5 shows a schematic of the active power control of the Type-3 WTG [19] whose output is the active current injection $I_{p \text { cmd }}$. Note that the active power control is regulated by three individual PI controllers as follows

Pitch Control: $G_{\text {cont }}(s)=K_{\mathrm{pp}}+\frac{K_{\mathrm{ip}}}{s}=150+\frac{25}{s}$

Pitch Comp.: $G_{\text {comp }}(s)=K_{\mathrm{pc}}+\frac{K_{\mathrm{ic}}}{s}=3+\frac{30}{s}$

Torque Control: $G_{\text {torq }}(s)=K_{\text {ptrq }}+\frac{K_{\text {itrq }}}{s}=3+\frac{0.6}{s}$

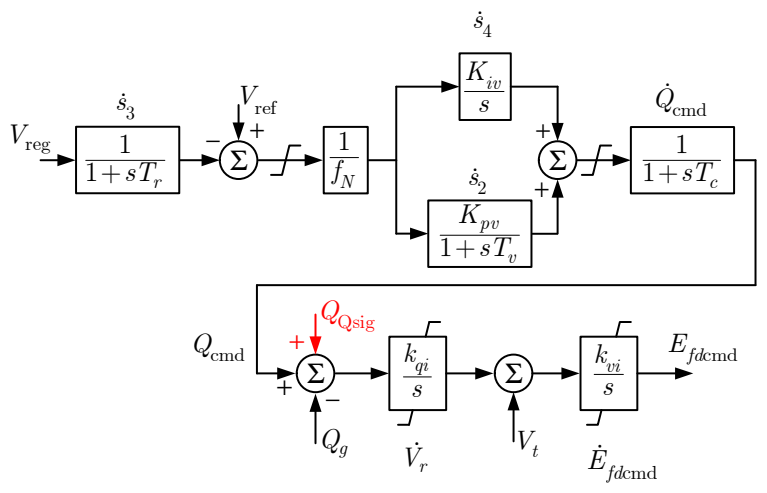

Figure 8: Schematic diagram of the reactive power control of the WTG model.

Fig. 8 illustrates the reactive power control of the Type-3 WTG [19]. Note that for this study the reactive power control of the WTG is set to control the voltage 
magnitude at the bus the WTG is connected to. Hence, this voltage signal corresponds to $V_{\text {reg. }}$. This function is called WindCONTROL and is a simplified version of the supervisory reactive power control of the entire wind farm [19].

\subsection{Control Design}

The control approach previously described is used for all combinations of input-output signals. Fig. 9 shows different root-locus plots of the test system $H_{\mathrm{xx}, \mathrm{zz}}(s)$ for all 12 combinations of input/output signals. These plots correspond to the power system with a specific pair of input and output signals as determined by the $B_{\mathrm{zz}}$ and $C_{\mathrm{xx}}$ matrices respectively. Because the correction of the departure angle comes only from the two stage lead-lag compensator, these plots also include the action of the washout filter. Columns in Fig. 9 determine the measured (output) signal while its rows correspond to the place to locate the controller in the WTG. The presence of zeros near the inter-area mode make some of the input/output signal combinations not suitable for the proposed controller. This situation however could be overcome by using multiple input signals to the controller [31] and robust control which are beyond the scope of this paper.

Out of the 12 possible controller combinations two controllers were selected. The first acts in the reactive power control of the WTG and uses the current magnitude as measured signal $G_{\mathrm{QQ}, \mathrm{Il}}$ while the second acts in the WTG active power setpoint using the bus frequency as feedback signal. The constants for the designed controllers are stated in Table 1. Fig. 10 shows a root-locus plot for these two controllers. These plots shows that the angle of departure is effectively near $180^{\circ}$ as intended and are used to select the appropriate proportional constant $K_{p}$. Fig. 11 shows the corresponding Bode plots to the Root Loci in Fig. 10. The frequency range of interest is from $0.1 \mathrm{~Hz}$ to $1 \mathrm{~Hz}$ because it is where inter-area oscillations occur. The Gain Margins of the the $G_{\mathrm{Pset}, \mathrm{f}}$ and $G_{\mathrm{QQ} \text {,Il }}$ controllers, respectively, is determined as 27.08 and $19.96 \mathrm{~dB}$.

Table 1: Designed Controllers Constants

\begin{tabular}{cccc}
\hline \hline Controller & $T_{1}[\mathrm{~s}]$ & $T_{2}[\mathrm{~s}]$ & $K_{p} \frac{\mathrm{pu}}{\mathrm{pu}}$ \\
\hline$G_{\mathrm{QQ}, \mathrm{Il}}$ & 1.1358 & 0.0824 & 0.4 \\
$G_{\text {Pset,f }}$ & 5.6613 & 0.0165 & 18 \\
\hline
\end{tabular}

This design was then evaluated when the WTG is connected to the system through a transmission line (TL) of different lengths. When increasing the length of the TL both controllers $\left(G_{\mathrm{P}_{\text {Set }} \text { f }}\right.$ and $\left.G_{\mathrm{QQ}, \mathrm{Il}}\right)$ become less effective. Because the connection between the WTG and the system becomes weaker it is also reasonable to
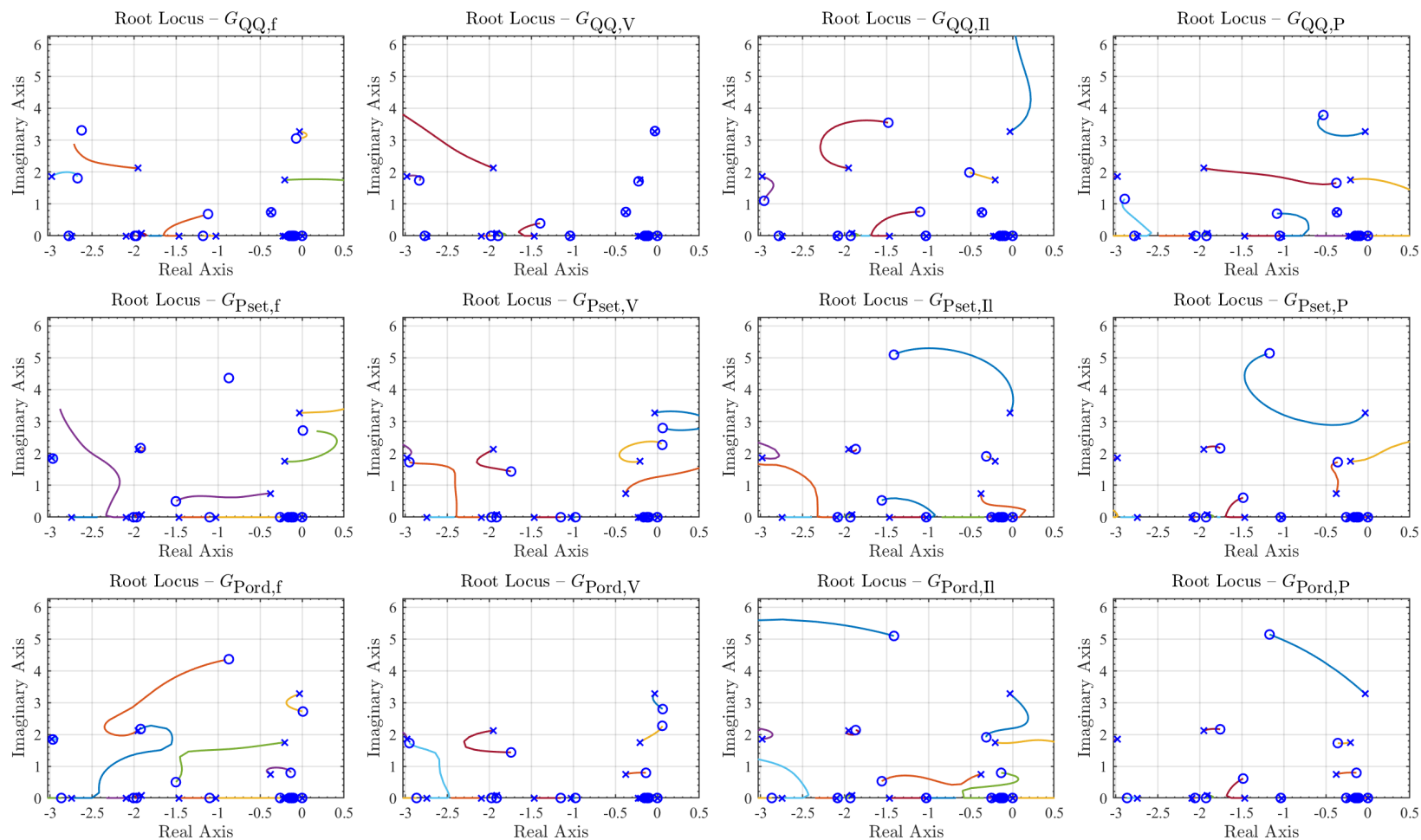

Figure 9: Root-loci for all possible combinations of input output signals. 


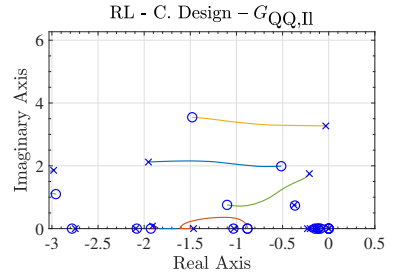

(a)

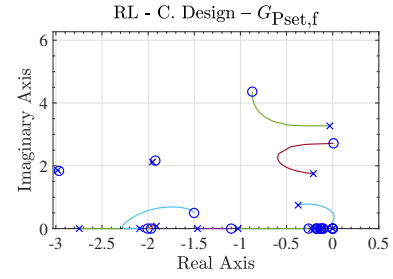

(b)
Figure 10: Root-loci for the two selected controllers: (a) $G_{\mathrm{QQ}, \mathrm{Il}}$, (b) $G_{\text {Pset,f }}$.
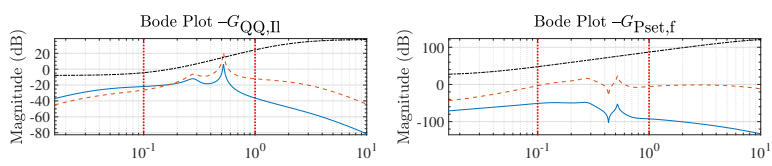

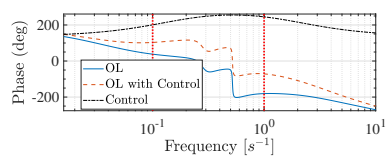

(a)

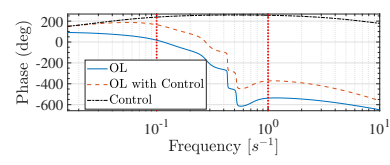

(b)
Figure 11: Bode plots for the two selected controllers: (a) $G_{\mathrm{QQ}, \mathrm{Il}}$, (b) $G_{\text {Pset,f. }}$.

expect the controller to be less effective.

Fig. 12 shows the zeros and the inter-area mode of the system as the TL increases from 0 to $100 \mathrm{~km}$ for the $G_{\mathrm{P}_{\mathrm{Set}}, \mathrm{f}}$ controller. For this same TL increase, the inter-area oscillation is slightly changed a result that is expected because the TL only connects the WTG to the system and does not significantly alter the characteristics of the inter-area tie line. By adjusting the controller time constants for optimal damping, the root-locus will result in the inter-area mode moving to the same zero for all TL lengths. Fig. 12 shows the movement of the dominant zero as the TL length increases; it shows that this zero approaches the inter-area mode of the system.

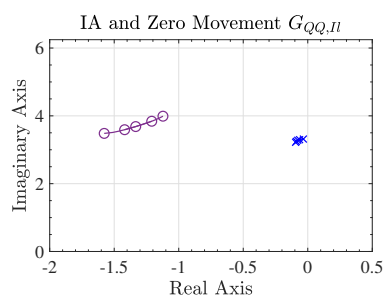

(a)

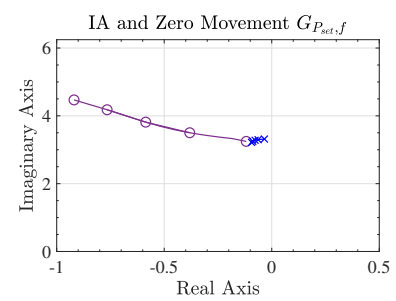

(b)
Figure 12: Zeros and Inter area mode with increasing TL length with: (a) $G_{\mathrm{QQ}, \mathrm{Il}}$, (b) $G_{\text {Pset,f }}$.

Similarly, Fig. 12b shows the zero and inter area movement of the $G_{\mathrm{QQ}, \text { Il }}$ controller. The poles of the system are the same as in the case of the $G_{\mathrm{P}_{\mathrm{Set}}, \mathrm{f}}$ controller. Although the zeros of the system differ a similar pattern emerges: The significant zero associated with the inter-area mode moves closer to the inter area mode as the TL length is increased. This indicates that both controllers become less effective with TL increases. Note that this effect is the result of having a zero moving towards the right hand plane and attracting the inter-area mode towards it for increases in the controller gain. Fig. 13b and Fig. 13a show the root-locus of the inter-area mode for each controller at 0,50 and $100 \mathrm{~km}$ TL length. These results show that the controllers are not able to significantly improve damping on the inter-area mode for the case of $100 \mathrm{~km}$ TL. In the $50 \mathrm{~km}$ TL case both controllers are capable of damping the inter-area mode better than the $100 \mathrm{~km}$ case. However, the effects of the controller and TL increases on the other modes of the system have to be considered. Fig. 14 shows the complete root locus for the case with a $100 \mathrm{~km}$ TL. Fig. 15 shows the corresponding Bode plots with the same frequency range of interest as in Fig. 11. From the bode plots and the Gain Margin can be determined as $21.67 \mathrm{~dB}$ and $25.29 \mathrm{~dB}$ for the $G_{\text {Pset,f }}$ and $G_{\mathrm{QQ}, \mathrm{Il}}$ controller respectively. To take into account all the modes of the system as well as important nonlinear phenomena, Section 5.2 presents time domain simulation results of the implemented controllers and analyzes their performance.

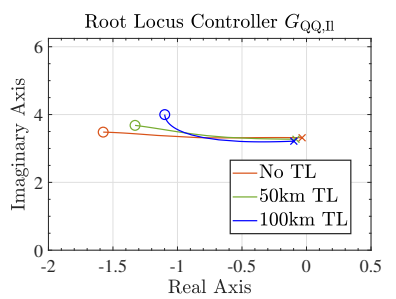

(a)

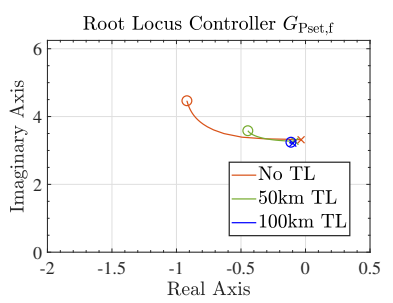

(b)
Figure 13: Root-loci for Controller design at different length TL for: (a) $G_{\mathrm{QQ}, \mathrm{Il}}$, (b) $G_{\text {Pset,f. }}$.

\section{Simulations Results}

This section presents time simulations results of the inter-area oscillation damping controllers of Section 4.

The test-system in Fig. 1 is selected to perform this analysis which is based on the following event: a three-phase short circuit to ground that lasts $0.1 \mathrm{~s}$ is applied to Bus 7 without changing the topology of the system once it is cleared.

The following two cases were considered when connecting the WTGs:

- The WTG is directly connected to the system using a step up transformer with $X=0.15 \mathrm{pu}$ in the machine base. This corresponds to a High Voltage connection of the WTG.

- The WTG is connected to the system through a $100 \mathrm{~km}$ transmission line (in addition to a 


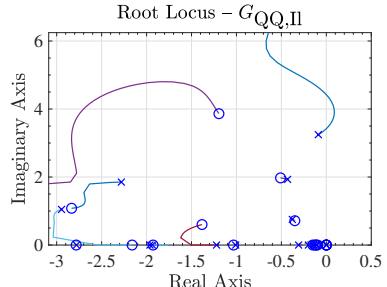

(a)

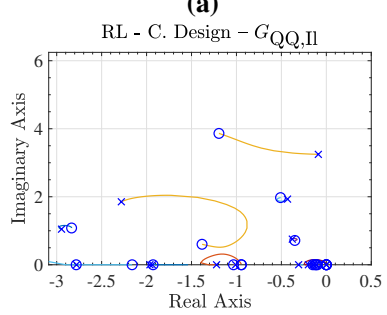

(c)

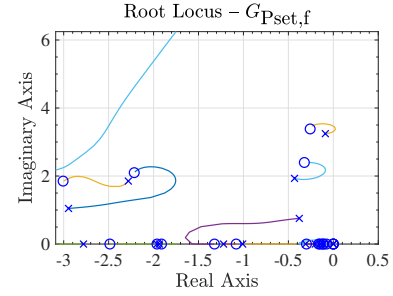

(b)

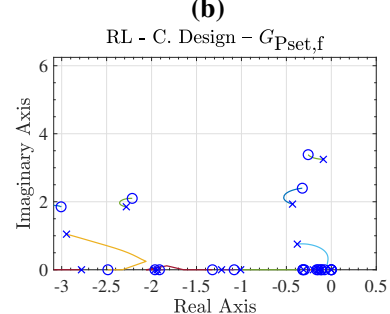

(d)
Figure 14: Root-loci for the two selected controllers with a long TL connecting the WTG to the system.
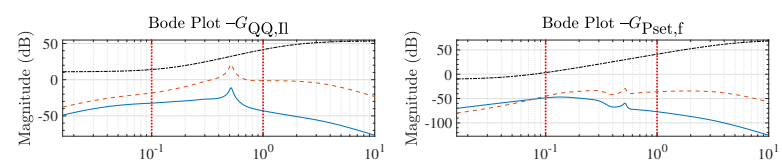

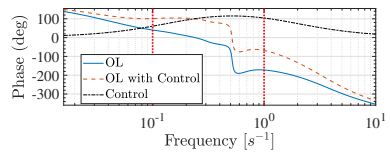

(a)

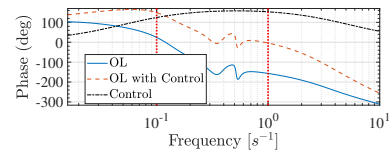

(b)
Figure 15: Bode plots for the two selected controllers with a long TL connecting the WTG to the system: (a) $G_{\mathrm{QQ}, \mathrm{Il}}$, (b) $G_{\text {Pset,f }}$.

similar transformer as in the previous case). This corresponds to a WTG located in a area with a weak grid connection.

\subsection{High Voltage Connection}

Four cases were considered: (i) a Type-3 WTG is installed in Area 1 producing around half of the total power in the area with the displaced conventional generation retaining the same MVA rating, (ii) similar to case (i) but including the $G_{\text {Pset,f }}$ controller of Section 4 to the WTG, (iii) similar to case (i) but including the $G_{\mathrm{QQ}, \mathrm{Il}}$ controller of Section 4 to the WTG and (iv) a no wind case for comparison.

Fig. 16 shows the results of the system for the cases (i)-(iv) just presented when Area 1 is importing 4 pu of real power as the loading condition of the system. The no wind case in these results show that the system has an inter-area mode that is excited by the applied fault and damped by the PSSs installed in conventional generators G2 and G4. They show that integrating wind in the area that is importing power (Area 1, Case A) reduces the damping of the power swing. They also show that when including any of the controllers $\left(G_{\text {Pset,f }}, G_{\mathrm{QQ}, \mathrm{Il}}\right)$ the inter-area oscillation is effectively damped as intended. Results in Fig. 16 (c) show that the controller $G_{\text {Pset,f }}$ makes the pitch angle responsive to the power swings. This result is expected because this controller is placed in the active power control loop of the WTG that is also responsible for managing the blade pitch. However it should be noted that this controller would be completely effective when the pitch angle has room for action (i.e., when the WTG is spilling some power). On the other hand, the controller $G_{\mathrm{QQ}, \mathrm{Il}}$ does not affect the pitch angle, it mainly affects the reactive power injection of the WTG which is reflected in the voltage behavior at Bus 6 as seen in Fig. 16 (b).

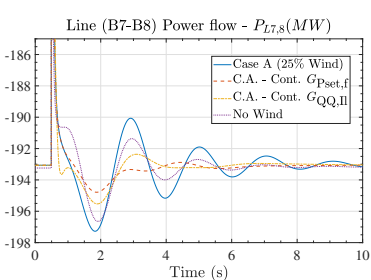

(a)

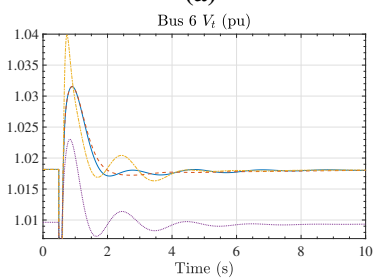

(c)

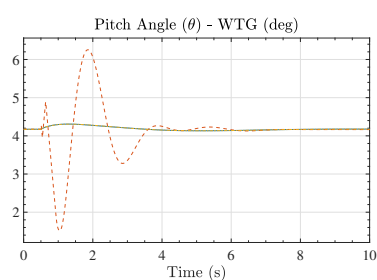

(b)

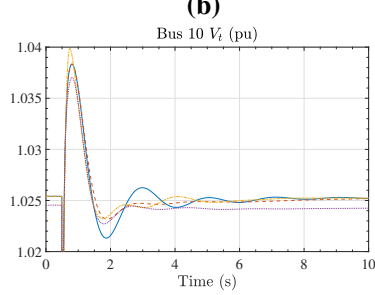

(d)
Figure 16: Plots for the case 4 pu of power is imported to Area 1. The following signals are presented for the different WTG control cases: (a) Power flow in line connecting Bus 7 and 8; (b) WTG pitch angle; (c) voltage at Bus 6; (d) voltage at Bus 10.

In addition, the two controllers were tested in a different power loading condition when Area 1 is exporting about $4 \mathrm{pu}$ to Area 2 and the results are presented in Fig. 17. These results show that both controllers are again effective in damping the inter-area oscillation $^{2}$. Note that for this case of power transfer wind integration in Area 1 also increases the damping of the power swing, a situation expected from the results outlined in Section 3.

Table 2 shows a summary of the inter-area mode for the different cases of wind integration and controller implementation in combination with the two power transfer conditions for a WTG with high Voltage connection.

\subsection{Low Voltage Connection}

The same four cases as in the high voltage connection case were considered in the low voltage connection case

\footnotetext{
${ }^{2}$ The gain of the controller for $G_{\mathrm{QQ}, \mathrm{Il}}$ has to be multiplied by -1 .
} 


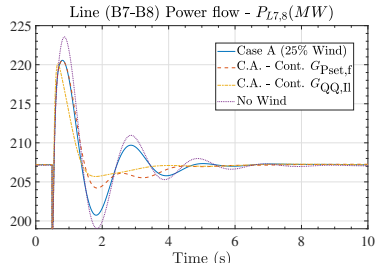

(a)

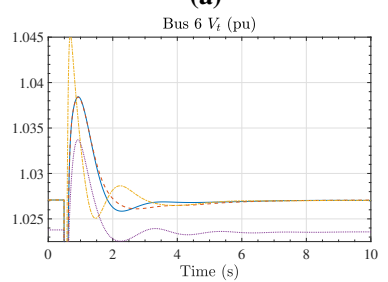

(c)

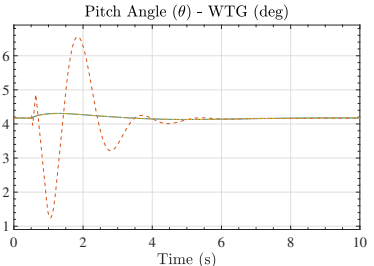

(b)

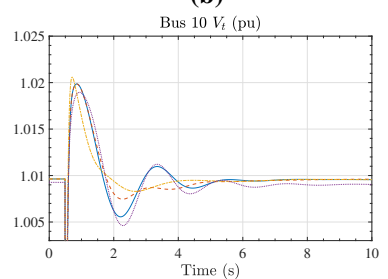

(d)

Figure 17: Plots for the case 4 pu of power is imported to Area 2. The following signals are presented for the different WTG control cases: (a) Power flow in line connecting Bus 7 and 8; (b) WTG pitch angle; (c) voltage at Bus 6; (d) voltage at Bus 10.

Table 2: Inter-area mode with controllers

\begin{tabular}{ccc}
\hline \hline Case & $P_{12}=-4 \mathrm{pu}$ & $P_{12}=4 \mathrm{pu}$ \\
\hline No wind & $-0.11+3.39 \mathrm{j}$ & $-0.13+3.39 \mathrm{j}$ \\
25\% Wind (Case A) & $-0.03+3.27 \mathrm{j}$ & $-0.42+3.39 \mathrm{j}$ \\
25\% W. (C. A) $-G_{\text {Pset,f }}$ & $-0.59+3.36 \mathrm{j}$ & $-0.65+3.61 \mathrm{i}$ \\
25\% W. (C. A) $-G_{\mathrm{QQ}, \mathrm{Il}}$ & $-0.34+3.28 \mathrm{j}$ & $-1.24+3.00 \mathrm{i}$ \\
\hline
\end{tabular}

where the TL length is set to $100 \mathrm{~km}$. Because the difference is the connection of the WTG, case (iv) which does not contain a WTG yields exactly the same results as in the previous section.

Fig. 18 shows the results of the system for cases (i)-(iv) when Area 1 is importing 4 pu of real power. Fig. 18 (c) shows that integrating wind in the area that is importing power (Area 1, Case A) reduces the damping of the power swing, even if the WTG is connected through a long TL. It also shows that any of the controllers $\left(G_{\text {Pset,f }}, G_{\mathrm{QQ}, \mathrm{Il}}\right)$ designed for a low voltage connection improves the inter-area oscillation damping to some degree.

Table 3 shows a summary of the inter-area mode for the different cases of controller implementation for a WTG connected to the system through a $100 \mathrm{~km}$ TL.

\section{Conclusions and Future Work}

This paper analyses the impact that integrating wind has on the inter-area oscillation of a test system. It presents a comprehensive study as to how this mode is affected by: (i) increased wind penetration for different WTG locations and (ii) increases in the reactance of the transmission line connecting the WTG to the system.

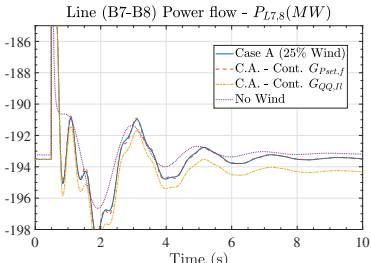

(a)

Bus $6 V_{t}(\mathrm{pu})$

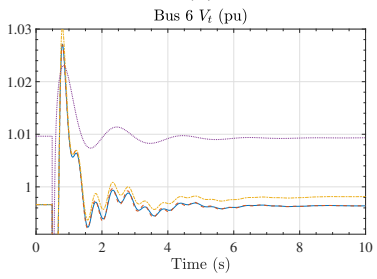

(c)

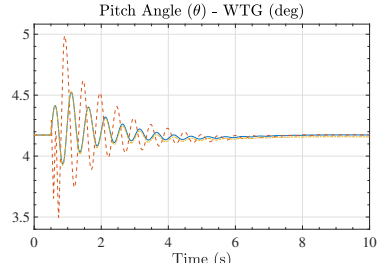

(b)

Bus $10 V_{t}(\mathrm{pu})$

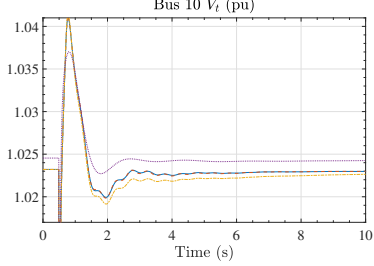

(d)
Figure 18: Plots for the case 4 pu of power is imported to Area 1 when the TL length is $100 \mathrm{~km}$. The following signals are presented for the different WTG control cases: (a) Power flow in line connecting Bus 7 and 8; (b) WTG pitch angle; (c) voltage at Bus 6; (d) voltage at Bus 10.

Table 3: Inter-area mode with controllers for the system with WTG connected through $100 \mathrm{~km}$ TL

\begin{tabular}{cc}
\hline \hline Case & $P_{12}=4 \mathrm{pu}$ \\
\hline No wind & $-0.1133+3.33868 \mathrm{j}$ \\
25\% Wind (Case A) & $-0.0958+3.2159 \mathrm{j}$ \\
25\% W. (C. A) $-G_{\text {Pset,f }}$ & $-0.1160+3.2545 \mathrm{j}$ \\
25\% W. (C. A) $-G_{\mathrm{QQ}, \mathrm{Il}}$ & $-0.3651+3.2878 \mathrm{j}$ \\
\hline
\end{tabular}

The paper shows that even though integrating wind integration in general increases the damping of the inter-area mode, it could potentially destabilize it when wind is installed in the area that imports power.

The paper then introduces a root-locus approach to design a two-stage lead lag controller for WTG to damp the inter-area oscillation of the system. Using this approach, two different controllers one acting in the active power control and the other in the reactive power structure of the WTG are designed and implemented. The paper then analyzes the effect on the controller performance of increases in the reactance of the transmission line that connects the WTG to the grid. Time domain simulation is used to test the performance of these controllers. These simulations show that wind can be effectively used to enhance the damping of the inter-area oscillations in power systems.

Future work will include designing a controller using more advanced multi-variable control techniques to deal with the zeros that appear near the inter-area mode when only SISO controllers are considered. 


\section{References}

[1] The Energy to Lead, 2015 New York State Energy Plan, New York State Energy Planning Board, Albany, NY, 2015.

[2] California Senate Bill SB-350 Clean Energy and Pollution Reduction Act of 2015, California Legislature, Sacramento, CA, 2015.

[3] J. Sanchez-Gasca, N. Miller, and W. Price, "A modal analysis of a two-area system with significant wind power penetration," in Power Systems Conf. and Expo., 2004. IEEE PES, New York, NY, 2004, pp. 1148-1152.

[4] G. Tsourakis, B. Nomikos, and C. Vournas, "Effect of wind parks with doubly fed asynchronous generators on small-signal stability," Electric Power Systems Research, vol. 79, no. 1, pp. 190-200, Jan. 2009.

[5] G. Tsourakis, B. M. Nomikos, and C. D. Vournas, "Contribution of doubly fed wind generators to oscillation damping," IEEE Trans. Energy Convers., vol. 24, no. 3, pp. 783-791, Sept. 2009.

[6] J. Morato, T. Knuppel, and J. Ostergaard, "Residue-based evaluation of the use of wind power plants with full converter wind turbines for power oscillation damping control," IEEE Trans. Sustain. Energy, vol. 5, no. 1, pp. 82-89, Jan. 2014.

[7] F. Wilches-Bernal, "Applications of wind generation for power system frequency control, inter-area oscillations damping and parameter identification," Ph.D. dissertation, Rensselaer Polytechnic Institute, 2015.

[8] C. Silva-Monroy, J. Neely, R. Byrne, R. Elliott, and D. Schoenwald, "Wind generation controls for damping of inter-area oscillations," in Power and Energy Soc. General Meeting, 2013 IEEE, Vancouver, BC, 2013, pp. $1-5$.

[9] L. Kunjumuhammed, B. Pal, K. Anaparthi, and N. Thornhill, "Effect of wind penetration on power system stability," in Power and Energy Soc. General Meeting, 2013 IEEE, Vancouver, BC, 2013, pp. 1-5.

[10] J.C. Neely et al., "Damping of inter-area oscillations using energy storage," in Power and Energy Soc. General Meeting, 2013 IEEE, Vancouver, BC, 2013, pp. 1-5.

[11] Z. Miao, L. Fan, D. Osborn, and S. Yuvarajan, "Control of DFIG-based wind generation to improve interarea oscillation damping," IEEE Trans. Energy Convers., vol. 24, no. 2, pp. 415-422, June 2009.

[12] D. Gautam, V. Vittal, R. Ayyanar, and T. Harbour, "Supplementary control for damping power oscillations due to increased penetration of doubly fed induction generators in large power systems," in Power Systems Conf. and Expo. (PSCE), 2011 IEEE/PES, Phoenix, AZ, 2011, pp. 1-6.

[13] F. M. Hughes, O. Anaya-Lara, N. Jenkins, and G. Strbac, "A power system stabilizer for DFIG-based wind generation," IEEE Trans. Power Syst., vol. 21, no. 2, pp. 763-772, May 2006.

[14] R. H. Byrne et al., "Small signal stability of the western north american power grid with high penetrations of renewable generation," in The 43rd Photovoltaic Specialist Conf. (PVSC), 2016. IEEE, 2016, pp. 1-6.

[15] F. Wilches-Bernal, J. H. Chow, and J. J. Sanchez-Gasca, "Impact of wind generation power electronic interface on power system inter-area oscillations," in Power and Energy Soc. General Meeting, 2016. IEEE, Boston, MA, 2016, pp. 1-5.

[16] F. Wilches-Bernal, C. Lackner, J. H. Chow, and J. J. Sanchez-Gasca, "Small-signal analysis of power system swing modes as affected by wind turbine-generators," in Power and Energy Conf. at Illinois (PECI), 2016 IEEE, Champaign, IL, 2016, pp. 1-6.
[17] J. H. Chow and K. W. Cheung, "A toolbox for power system dynamics and control engineering education and research," IEEE Trans. Power Syst., vol. 7, no. 4, pp. 1559-1564, Nov. 1992.

[18] F. Wilches-Bernal, J. J. Sanchez-Gasca, and J. H. Chow, "Implementation of wind turbine generator models in the power system toolbox," in Power and Energy Conf. at Illinois (PECI), 2014 IEEE, Champaign, IL, 2014, pp. $1-5$.

[19] M. Shao, N. Miller, J. Sanchez-Gasca, and J. MacDowell, "Modeling of GE Wind Turbine-Generator for Grid Studies," General Electric International, Inc., Schenectady, NY, Tech. Rep. Version 4.6, Mar. 2013.

[20] CIGRE Technical Brochure 318: Modeling and Dynamic Behavior of Wind Generation as it Relates to Power System Control and Dynamic Performance, CIGRE, Paris, France, Aug. 2007.

[21] N. Miller et al., "Coordinated voltage control for multiple wind plants in Eastern Wyoming: Analysis and field experience," in Power Electronics and Machines in Wind Applications (PEMWA), 2012 IEEE, Denver, CO, 2012, pp. 1-8.

[22] J. M. MacDowell, K. Clark, N. W. Miller, and J. J. Sanchez-Gasca, "Validation of GE wind plant models for system planning simulations," in Power and Energy Soc. General Meeting, 2011 IEEE, Detroit, MI, 2011, pp. 1-8.

[23] N. Miller, R. Delmerico, K. Kuruvilla, and M. Shao, "Frequency responsive controls for wind plants in grids with wind high penetration," in Power and Energy Soc. General Meeting, 2012 IEEE, San Diego, CA, 2012, pp. $1-7$.

[24] N. Miller, M. Shao, S. Pajic, and R. D'Aquila, "Eastern frequency response study," National Renewable Energy Laboratory (NREL), Golden, CO., Tech. Rep. NREL/SR-5500-58077, May 2013.

[25] M. Asmine et al., "Model validation for wind turbine generator models," IEEE Trans. Power Syst., vol. 26, no. 3, pp. 1769-1782, Aug. 2011.

[26] P. Kundur, Power System Stability And Control, ser. EPRI power system engineering series. McGraw-Hill, 1994. [Online]. Available: https://books.google.com/books?id= v3RxH $\backslash$ _GkwmsC

[27] E. Larsen and D. Swann, "Applying power system stabilizers part I: General concepts," IEEE Trans. Power App. Syst., vol. PAS-100, no. 6, pp. 3017-3024, June 1981.

[28] E. Larsen and D. Swann, "Applying power system stabilizers part II: Performance objectives and tuning concepts," IEEE Trans. Power App. Syst., vol. PAS-100, no. 6, pp. 3025-3033, June 1981.

[29] J. H. Chow, G. E. Boukarim, and A. Murdoch, "Power system stabilizers as undergraduate control design projects," IEEE Trans. Power Syst., vol. 19, no. 1, pp. 144-151, Feb. 2004.

[30] A. Murdoch and G. Boukarim, "Performance criteria and tuning techniques," in IEEE Tutorial Course on Power System Stabilization Via Excitation Control, Tampa, FL: IEEE, 2007, ch. 3, pp. 26-34.

[31] J. H. Chow, J. J. Sanchez-Gasca, H. Ren, and S. Wang, "Power system damping controller design using multiple input signals," IEEE Control Syst. Mag., vol. 20, no. 4, pp. 82-90, Aug. 2000. 